\title{
Effect of a Single Dislocation in a Heterostructure Layer on the Local Polarization of a Ferroelectric Layer
}

\author{
C. L. Jia, ${ }^{1}{ }^{*}$ S. B. Mi, ${ }^{1}$ K. Urban, ${ }^{1}$ I. Vrejoiu, ${ }^{2}$ M. Alexe,${ }^{2}$ and D. Hesse ${ }^{2}$ \\ ${ }^{1}$ Institute of Solid State Research and Ernst Ruska-Centre for Microscopy and Spectroscopy with Electrons (ER-C), \\ Forschungszentrum Jülich, D-52425 Jülich, Germany \\ ${ }^{2}$ Max Planck Institute of Microstructure Physics, Weinberg 2, D-06120 Halle, Germany
}

(Received 12 September 2008; published 16 March 2009)

\begin{abstract}
We study, on an atomic scale, the influence of a single dislocation in a $\mathrm{SrTiO}_{3}$ sublayer on the local ferroelectric polarization of the neighboring ferroelectric $\mathrm{PbZr}_{0.2} \mathrm{Ti}_{0.8} \mathrm{O}_{3}(\mathrm{PZT})$ sublayer in an epitaxial $\mathrm{SrTiO}_{3} / \mathrm{PbZr}_{0.2} \mathrm{Ti}_{0.8} \mathrm{O}_{3} / \mathrm{SrTiO}_{3}$ three-layer heterostructure. The strain field of the dislocation in the $\mathrm{SrTiO}_{3}$ layer propagates across the interface into the PZT layer and leads to a strong variation of the $c$-lattice parameter of the PZT layer. Accompanying a strong reduction of the $c$-lattice parameter, the offcenter displacements of the $\mathrm{Zr} / \mathrm{Ti}$ atoms away from the center of the oxygen octahedra are also strongly decreased, resulting in a decrease of the local spontaneous polarization by up to $48 \%$.
\end{abstract}

DOI: 10.1103/PhysRevLett.102.117601

Owing to their excellent properties, perovskite ferroelectrics have become important materials in electronics applications [1-3]. In more complex systems like perovskite ferroelectric-paraelectric superlattices or tricolor superlattices intriguing properties and effects arise due to the combination of materials with similar structure [4,5]. Adjusting the composition/periodicity in these superlattices allows for an additional degree of freedom and results in the possibility to tune the final properties. As has recently been shown [6], a classical ferroelectric-paraelectric system, such as $\mathrm{PbTiO}_{3} / \mathrm{SrTiO}_{3}$ can, under certain conditions, behave like an improper ferroelectric only due to a ferroelectric/antiferrodistortive coupling at the interface between the two materials. In experimental and theoretical analysis of these effects one usually assumes that one is dealing with perfect materials. Oxides and, in particular, ferroelectric perovskite oxides are, however, far from being perfect materials. When the characteristic sizes of the investigated systems are scaling down to nanometer sizes even a single lattice defect can be very detrimental to ferroelectric properties. An example is the case of dislocations that are not avoidable in most crystalline materials. In the surrounding area a dislocation produces strain which depends on the Burgers vector of the dislocation and the location with respect to the dislocation core [7].

The effect of dislocation strain fields on the local ferroelectric polarization has been investigated theoretically $[8,9]$ in ferroelectric $\mathrm{PbZr}_{1-x} \mathrm{Ti}_{x} \mathrm{O}_{3}$. It was demonstrated that the coupling between the strain field of the dislocation and the polarization results in high local polarization gradients, leading to strong depolarization fields within a distance of about $10 \mathrm{~nm}$ from the dislocation core. In experiment, a high density of threading dislocations occurring in epitaxial $\mathrm{PbZr}_{0.2} \mathrm{Ti}_{0.8} \mathrm{O}_{3}$ films has been reported to affect the ferroelectric switching properties [10]. A study on epitaxial $\mathrm{PbZr}_{0.52} \mathrm{Ti}_{0.48} \mathrm{O}_{3}$ nanoislands on $\mathrm{Nb}$-doped $\mathrm{SrTiO}_{3}$ substrate has related a poor piezoresponse to the
PACS numbers: 77.84.Bw, 61.72.Ff, 68.37.Og, 77.22.Ej

strain field resulting from interfacial dislocations [11]. A study on the role of misfit dislocations in destabilizing the polar state was reported in epitaxial ferroelectric thin films [12]. However, a genuine measurement of polarization change in a dislocation field has so far not been carried out. The atomic-resolution study of the behavior of a unit cell dipole near a dislocation requires direct imaging or determining the atomic structure at subangstrom accuracy $[13,14]$.

Here we employ the negative $C_{S}$ imaging (NCSI) technique [15] to study the change of the local polarization in a ferroelectric $\mathrm{PbZr}_{0.2} \mathrm{Ti}_{0.8} \mathrm{O}_{3}$ (PZT) layer due to the presence of a single dislocation in the neighboring dielectric $\mathrm{SrTiO}_{3}$ (STO) layer, in a model sample consisting of three epitaxial layers of STO/PZT/STO, all prepared by pulsed laser deposition on a STO (100) substrate [16].

Figure 1(a) shows an image of an interface area between the bottom STO layer and the $c$ axis oriented, tetragonal PZT layer at a low magnification. The image is recorded with electron beam parallel to the [110] direction of both compounds under the NCSI conditions. The interface is marked by a dotted line. A dislocation is denoted in the lower-left part of the STO layer by a symbol " $\perp$ ". A Burgers circuit surrounding the dislocation core leads to a closure failure with a projected displacement vector $a\left[\frac{1}{2} \frac{1}{2} \overline{1}\right]$ along the [110] direction. Since no other defects are found to accompany the dislocation, the real Burgers vector must include a component along the viewing direction. This consideration leads to the possible Burger vector either $a[011]$ or $a[101]$. In the core area of the dislocation the image contrast is smeared out due to a short-distance dissociation of the dislocation and strong lattice distortion. Above the dislocation core the (001) planes bend upward as shown by a curved open arrow. The upward bending of the lattice plane is seen to propagate through the interface into the PZT film area, resulting in a compressive stress along the $c$ axis of the PZT film. This bending gets weaker 


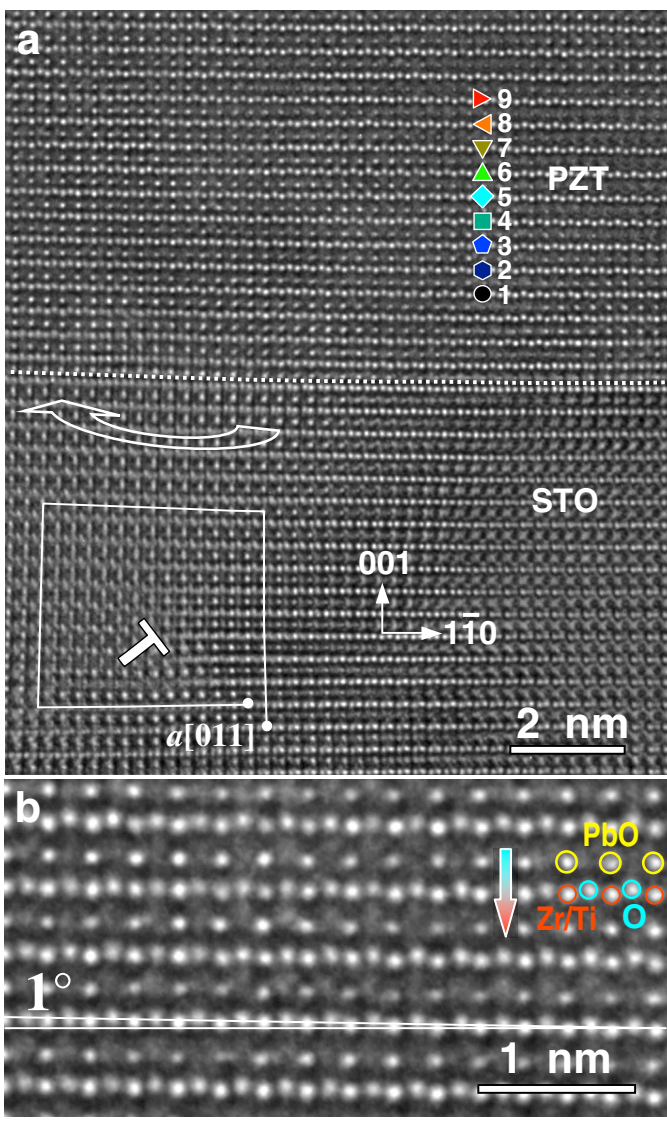

FIG. 1 (color online). (a) Atomic-resolution [110] image of part of a STO/PZT/STO heterostructure including a dislocation denoted by " $\perp$ ", recorded under NCSI conditions. The interface between the bottom STO and PZT layers is marked by a dotted line. The curved arrow shows the strong upward bending of the (001) planes. The numbered symbols mark the (001) $(\mathrm{Zr} / \mathrm{Ti}) \mathrm{O}_{2}$ planes for quantitative analysis. (b) A magnification of an area directly above the interface in (a). The columns of $\mathrm{PbO}, \mathrm{Zr} / \mathrm{Ti}$ and $\mathrm{O}$ are denoted. The relative displacements between positively charged cations $(\mathrm{Pb}, \mathrm{Zr} / \mathrm{Ti})$ and negatively charged anion (O) result in an electric dipole in the unit cell and define the spontaneous polarization vector as show by an arrow. Two lines trace the lattice bending from left to the right of the image, which amounts to about one degree.

as going from the left to the right, i.e., as going from the area right above the core to the area far from the core. Figure 1(b) shows a magnification of a PZT film area above the interface. In the NCSI image the atoms appear bright on a dark background. The $\mathrm{PbO}, \mathrm{Zr} / \mathrm{Ti}$, and $\mathrm{O}$ columns are marked by circles. The shifts of the oxygen atoms are evidently directed upward, resulting in an upward shift of the negative charge center in the unit cell. As a consequence, the polarization in this area points downward, from the net negative charge to the net positive charge in the unit cell.

Various properties related to the bending of the lattice planes in the PZT layer are studied by quantitatively mapping the image in the area from the left edge (zero position) to the place marked by a group of circles where the lattice bending is seen to vanish in Fig. 1(a). In order to minimize the effects of the contrast noise, before mapping the image is filtered in such a way that the quantitative results should not be affected [13]. In image mapping the positions of the bright dots, which correspond to the atom columns, are determined using a least square fit by two-dimensional Gaussian profiles to each of the individual atomic intensity profiles [17]. All of the measurements are based on the positions of the intensity maxima corresponding to the oxygen and the $\mathrm{Zr} / \mathrm{Ti}$ columns. The upward bending of lattice planes is first investigated by checking the relative vertical position of the individual unit cells. The relative positions are presented by the relative vertical shifts $\Delta_{j}\left(d_{i}\right)$ of individual $\mathrm{Zr} / \mathrm{Ti}$ columns in a given $(001)(\mathrm{Zr} / \mathrm{Ti}) \mathrm{O}_{2}$ plane $j(j=1-9$, marked by a group of symbols). In a given $j$ plane the values of $\Delta_{j}\left(d_{i}\right)$ are defined by $\Delta_{j}\left(d_{i}\right)=$ $Z_{j}\left(d_{i}\right)-Z_{j}(\infty)$, where $Z_{j}\left(d_{i}\right)$ is the vertical coordinate of the $\mathrm{Zr} / \mathrm{Ti}$ column No. $i$ at a distance $d_{i}$ from the left edge of the image in Fig. 1(a) and $Z_{j}(\infty)$ is the mean of the vertical coordinates of the $\mathrm{Zr} / \mathrm{Ti}$ columns at the area of the symbol group. Figure 2 displays the values of $\Delta_{j}\left(d_{i}\right)$ as a function of the distance $d_{i}$. It is evident that the shifts of the $\mathrm{Zr} / \mathrm{Ti}$ columns in planes $j=1-6$ decrease when the columns are going from left to the right and reach essentially zero in the area relatively far from the dislocation core (near the position of the symbol group).

Based on the coordinates of the $\mathrm{Zr} / \mathrm{Ti}$ columns the $c$-lattice parameter for individual unit cells is calculated and calibrated referring to the lattice constant $(a=$ $0.3905 \mathrm{~nm}$ ) of $\mathrm{SrTiO}_{3}$. We note that depolarization fields can be significant in a ferroelectric thin film on an insulating substrate $[18,19]$. The presence of a depolarization field may result in rather complex polarization patterns near the interface. These may imply vertical and lateral gradients, especially when $180^{\circ}$ stripe domains are present. As a consequence, the local spontaneous polarization and the $c$-lattice parameter of the unit cells can be changed $[14,20]$. In our specific case the mapped area is far from

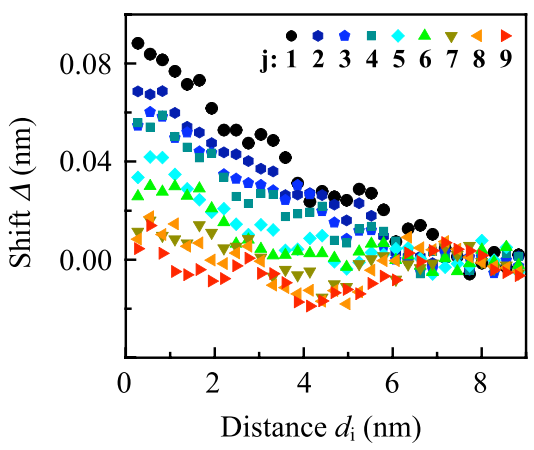

FIG. 2 (color online). Lattice plane bending in the PZT layer as a function of the distance from the left edge of the image in Fig. 1(a). The bending is represented by the vertical shifts $\Delta$ of the $\mathrm{Zr} / \mathrm{Ti}$ columns in a given $(001)(\mathrm{Zr} / \mathrm{Ti}) \mathrm{O}_{2}$ plane $(j=1-9)$ with respect to the $\mathrm{Zr} / \mathrm{Ti}$ columns in the perfect area at the position of the symbol group in Fig. 1(a). 
domain walls. In the studied area no $180^{\circ}$ stripe domains and other complex polarization patterns are found employing the NCSI imaging technique which allows any lateral or vertical polarization gradients to be directly visualized [13]. Therefore, the effect of lateral gradient can be neglected. In addition, inhomogeneous strain can exist in a film due to the lattice mismatch between STO and PZT, which depends on the location with respect to the interfaces [21]. Such effects of the vertical gradient on the studied parameters can be mitigated by averaging the values of the measured parameters along the normal of the interface, concentrating on the effect of the dislocation. This results in the observation of the dependence of the $c$-lattice parameter as a function of the lateral distance from the left edge of the image, i.e., from the area near the dislocation core. The mean values of the $c$-lattice parameters are calculated by averaging over 6 unit cells along the [001] direction. Figure 3(a) displays the $c$-lattice parameter as a function of the distance from the left edge. A continuous increase of the $c$ value is evident from about $0.405 \mathrm{~nm}$ at the left edge of the image to about $0.416 \mathrm{~nm}$ representing the value in the perfect film matrix at the position of the circle group. At the area right above the dislocation core the value of the lattice parameter $c$ is reduced by about $3 \%$.

Accompanying the reduction in $c$-lattice parameter, the reduction of the vertical spacing $\delta_{\mathrm{O}-\mathrm{Zr} / \mathrm{Ti}}$ between the oxygen columns and the neighboring $\mathrm{Zr} / \mathrm{Ti}$ columns is also measured. Figure 3(b) displays the vertical spacing $\delta_{\mathrm{O}-\mathrm{Zr} / \mathrm{Ti}}$ as a function of the distance from the left edge of image in Fig. 1(a). In the perfect area at the position of the circle group the value of the spacing is about $0.0296 \mathrm{~nm}$ while in the area right above the dislocation core near the left edge of the image the minimum value is about $0.0166 \mathrm{~nm}$. The maximum reduction of the vertical spacing is about $44 \%$ in the mapped area. Since the spontaneous polarization $P_{S}$ arises from the electric dipole which forms by the relative displacements of ions in the unit cells, the dependence of the $\mathrm{O}-\mathrm{Zr} / \mathrm{Ti}$ spacing on the distance from the dislocation shows also evidence for the dependence of the local polarization.

The spontaneous polarization can be derived from $c$-lattice parameters based on Landau-GinzburgDevonshire theory by the formula [14]

$$
P=\left(\frac{x_{3}-2 s_{12} x_{m} /\left(s_{11}+s_{12}\right)}{Q_{11}-2 s_{12} Q_{12} /\left(s_{11}+s_{12}\right)}\right)^{1 / 2},
$$

where $x_{3}$ and $x_{m}$ are the spontaneous strains defined by $x_{3}=\left(c-a_{p}\right) / a_{p}$ and $x_{m}=\left(a-a_{p}\right) / a_{p}\left(a_{p}\right.$ is the lattice parameter of the paraelectric cubic phase extrapolated to room temperature), and $s_{i j}$ and $Q_{i j}$ are elastic compliances and electrostrictive coefficients, respectively. The $c$ values are the measured values shown in Fig. 3(a) and the $a$ value is the lattice parameter of the STO substrate. The values of $a_{P}, s_{i j}$, and $Q_{i j}$ for PZT are adopted from Refs. [22-24]. The calculated result is presented by the circles in Fig. 3(c).

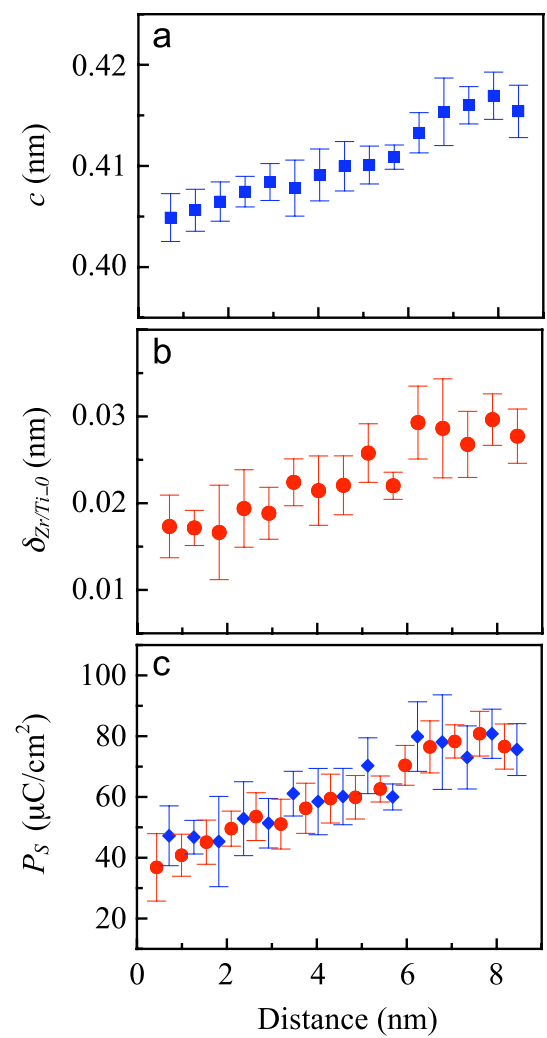

FIG. 3 (color online). $\quad c$-lattice parameter (a), vertical spacing $\delta_{\mathrm{O}-\mathrm{Zr} / \mathrm{Ti}}$ between $\mathrm{O}$ and $\mathrm{Zr} / \mathrm{Ti}$ columns (b) and spontaneous polarization (c) of the PZT layer as a function of the distance from the left edge of the image in Fig. 1(a). In (c) circles display the result calculated from relation (1) and diamonds from (3). The error bars represent the mean error with a confidence level of $95 \%$.

The local spontaneous polarization $P_{S}$ can also be calculated based on the off-center displacement of atoms in the unit cell by the relation [14]:

$$
P_{S}=1 / \nu \sum Z_{i} \Delta_{i}
$$

where $\nu, \Delta_{i}$, and $Z_{i}$ are the volume of the unit cell, the offcenter displacement of atom $i$, and the effective charge of atom $i$, respectively. We note that we are directly measuring the spacing between the oxygen column and the $\mathrm{Zr} / \mathrm{Ti}$ column. The spacing represents the displacement of the $\mathrm{Zr} / \mathrm{Ti}$ column from the center of the oxygen octahedron if we neglect the small distortion of the oxygen octahedron. Based on the data in hand [Fig. 3(b)] the local spontaneous polarization $P_{S}$ is roughly estimated using a linear relation [25]:

$$
P_{S}=\kappa \delta_{\mathrm{Zr} / \mathrm{Ti}}
$$

where $\kappa$ is a constant and $\delta_{\mathrm{Zr} / \mathrm{Ti}}$ is the displacement of the $\mathrm{Zr} / \mathrm{Ti}$ column from the center of the oxygen octahedron. In calculation of $P_{S}$ a value of $\kappa$ of $2726\left(\mu \mathrm{C} / \mathrm{cm}^{2}\right) \mathrm{nm}^{-1}$ [20] is used. Based on the data of atom displacements in the same film system published in [13], a test to evaluate the 
deviation of the $P_{S}$ values calculated using relation (3) from those calculated from relation (2) leads to the absolute values $<10 \mu \mathrm{C} / \mathrm{cm}^{2}$ of the deviation. For comparison, the polarization calculated from (3) is presented by diamonds in Fig. 3(c). The result calculated from (3) agrees well with that from (1). An increase of the local polarization is evidently with the distance from the dislocation core. Considering the mean values of the results from (1) and (3), the value of $P_{S}$ is about $80 \mu \mathrm{C} / \mathrm{cm}^{2}$ in the area far from the dislocation core. In the area directly above the dislocation core the value of $P_{S}$ is about $42 \mu \mathrm{C} / \mathrm{cm}^{2}, 52 \%$ of the value in the defect-free area.

Figure 3 provides evidence for the effect of the dislocation on the local properties of the PZT layer. In addition, we estimate the possible errors created by residual lens aberrations, local change of sample thickness, and small (unavoidable) tilts away from the fully symmetric Laue orientation. This is achieved by carrying out an iterative procedure in which the experimental image is compared with images calculated on a computer following the established procedures $[13,26]$. The estimation shows that the errors are less than $\pm 0.002 \mathrm{~nm}$ for the vertical spacing between the oxygen and $\mathrm{Zr} / \mathrm{Ti}$ columns, much smaller than the mean error bars (about $\pm 0.005 \mathrm{~nm}$ ) shown in Fig. 3(b).

In the ideal case of a dislocation located at the center of a perfect lattice matrix, the strain distribution is expected to be symmetric with respect to the extra half plane. In the reality of the TEM specimen, this symmetric feature is broken by the off-central location of the dislocation, the inhomogeneity of the specimen thickness, and difference in mechanical properties of the heterostructure layers. Therefore, the measured various properties in real specimens may deviate from those expected for the ideal case. Nevertheless, the asymmetry of real specimens does not principally influence the result obtained on the effect of a single dislocation, though distant from the ferroelectric layer, onto the local polarization of the latter. In addition to the effect of strain, we note that a possible charge of the dislocation core can be induced by local chemical deviations from the ideal stoichiometry. A deficiency of oxygen can, e.g., result in a positive charge of the dislocation core $[27,28]$. The positive charges at the dislocation site create a depolarization field with respect to the polarization domain in the affected region of the PZT layer. The observed strong bending of the STO lattice planes at the interface, which is not expected to be affected by the electric field, shows that the lattice strain propagates into the neighboring PZT layer and thus plays a major role in depressing the spontaneous polarization. A recent study showed that a strain gradient can induce a polarization in a STO crystal [29]. A local polarization of about $1-10 \mu \mathrm{C} / \mathrm{cm}^{2}$ was estimated around a dislocation in STO at room temperature. The flexoelectric polarization induced by the strain field of the dislocation may also produce a depolarization field with respect to the polarization domain of the PZT layer.
Our experimental study shows, on atomic scale, the behavior of unit cell dipoles of ferroelectric PZT in the field of a dislocation. In a region of the PZT layer about $8 \mathrm{~nm}$ away from the dislocation core a strong reduction of the $c$-lattice parameter is measured. A corresponding strong reduction of the off-center displacements of the $\mathrm{Zr} / \mathrm{Ti}$ atoms reflects the strain-polarization coupling of the ferroelectric material. This study demonstrates that a single lattice defect, such as a dislocation, even though it is located well outside the ferroelectric material, can have a detrimental effect on the properties of the ferroelectric layer.

*c.jia@fz-juelich.de

[1] N. Setter and R. Waser, Acta Mater. 48, 151 (2000).

[2] M. Dawber, K. M. Rabe, and J. F. Scott, Rev. Mod. Phys. 77, 1083 (2005).

[3] J. F. Scott, Science 315, 954 (2007).

[4] H. N. Lee et al., Nature (London) 433, 395 (2005).

[5] M. Dawber et al., Phys. Rev. Lett. 95, 177601 (2005).

[6] E. Bosquet et al., Nature (London) 452, 732 (2008).

[7] J. P. Hirth and J. Lothe, Theory of Dislocations (McGrawHill, New York, 1968).

[8] S. P. Alpay, I. B. Misirlioglu, V. Nagarajan, and R. Ramesh, Appl. Phys. Lett. 85, 2044 (2004).

[9] I. B. Misirlioglu, G. Akcay, and S.P. Alpay, Integr. Ferroelectr. 83, 67 (2006).

[10] I. Vrejoiu et al., Philos. Mag. 86, 4477 (2006).

[11] M. W. Chu et al., Nature Mater. 3, 87 (2004).

[12] V. Nagarajan et al., Appl. Phys. Lett. 86, 192910 (2005).

[13] C. L. Jia et al., Nature Mater. 7, 57 (2008).

[14] D. D. Fong et al., Phys. Rev. B 71, 144112 (2005).

[15] C. L. Jia, M. Lentzen, and K. Urban Science 299, 870 (2003).

[16] I. Vrejoiu et al., Adv. Mater. 18, 1657 (2006).

[17] L. Houben, A. Thust, and K. Urban, Ultramicroscopy 106, 200 (2006).

[18] G. B. Stephenson and K. R. Elder, J. Appl. Phys. 100, 051601 (2006).

[19] S. Prosandeev and L. Bellaiche, Phys. Rev. B 75, 172109 (2007).

[20] C. L. Jia et al., Nature Mater. 6, 64 (2007).

[21] G. Catalan et al., Phys. Rev. B 72, 020102 (2005).

[22] S. P. Alpay, V. Nagarajan, L. A. Bendersky, M. D. Vaudin, S. Aggarwal, R. Ramesh, and A. L. Roytburd, J. Appl. Phys. 85, 3271 (1999).

[23] N. A. Pertsev, V. G. Kukhar, H. Kohlstedt, and R. Waser, Phys. Rev. B 67, 054107 (2003).

[24] M. J. Haun, Z. Q. Zhuang, E. Furman, S. J. Jang, and L. E. Cross, Ferroelectrics 99, 45 (1989).

[25] S. C. Abrahams S. K. Kurtz, and P. B. Jamieson, Phys. Rev. 172, 551 (1968).

[26] K. Urban, Science 321, 506 (2008).

[27] Z. Zhang, W. Sigle, and M. Rühle, Phys. Rev. B 66, 094108 (2002).

[28] C. L. Jia, A. Thust, and K. Urban, Phys. Rev. Lett. 95, 225506 (2005).

[29] P. Zubko et al., Phys. Rev. Lett. 99, 167601 (2007). 